\title{
Effect of Cell Seeding on Neotissue Formation in a Tissue Engineered Trachea
}

Elizabeth S. Clark, ${ }^{\mathrm{a}, \mathrm{b}}$ Cameron Best, ${ }^{\mathrm{a}}$ Ekene Onwuka, ${ }^{\mathrm{a}, \mathrm{c}}$ Tadahisa Sugiura, ${ }^{\mathrm{a}}$ Nathan Mahler, ${ }^{\mathrm{a}}$ Brad Bolon, ${ }^{\mathrm{b}, \mathrm{d}}$ Andrew Niehaus, ${ }^{\text {e }}$ Iyore James, ${ }^{a}$ Narutoshi Hibino, ${ }^{\text {a,f }}$ Toshiharu Shinoka, ${ }^{\text {a,f }}$ Jed Johnson, ${ }^{\mathrm{g}}$ Christopher K. Breuer ${ }^{\mathrm{a}, \mathrm{h}}$

\author{
Affiliations: \\ a Tissue Engineering and Surgical Research, The Research Institute at Nationwide Children's Hospital, 700 \\ Children's Drive - Suite WB4154, Columbus, OH, 43205 \\ ${ }^{\mathrm{b}}$ Department of Veterinary Biosciences, College of Veterinary Medicine, The Ohio State University, 1900 Coffey \\ Road, Columbus, OH, 43210 \\ ${ }^{\mathrm{c}}$ Department of Surgery, The Ohio State University, 395 W. $12^{\text {th }}$ Avenue - Suite 670, Columbus, OH, 43210 \\ ${ }^{\mathrm{d}}$ Comparative Pathology and Mouse Phenotyping Shared Resource, College of Veterinary Medicine, The Ohio \\ State University, 1900 Coffey Road, Columbus, OH, 43210 \\ ${ }^{\mathrm{e}}$ Department of Veterinary Clinical Sciences, College of Veterinary Medicine, The Ohio State University, 601 \\ Vernon Tharp Street, Columbus, OH, 43210 \\ ${ }^{\mathrm{f}}$ Department of Cardiothoracic Surgery, Nationwide Children's Hospital, 700 Children's Drive, Columbus, OH, \\ 43205 \\ ${ }^{g}$ Nanofiber Solutions, Inc., 1275 Kinnear Road, Columbus, OH, 43212 \\ h Department of Pediatric Surgery, Nationwide Children's Hospital, 700 Children's Drive, Columbus, OH, 43205
}

\section{Corresponding Author:}

Christopher K. Breuer, MD

Director, Tissue Engineering and Surgical Research

The Research Institute at Nationwide Children's Hospital

700 Children's Drive - WB 4151

Columbus, $\mathrm{OH} 43205$

Phone: 614.355.5754

E-mail: Christopher.Breuer@NationwideChildrens.org 


\section{Author Roles:}

Elizabeth S. Clark: Study design, acquisition, analysis, and interpretation of data, manuscript preparation Cameron Best: Study design, acquisition and analysis of data, surgical assistant, manuscript preparation Ekene Onwuka: Analysis and interpretation of data, manuscript preparation

Tadahisa Sugiura: Acquisition of in vitro data, primary surgeon, manuscript preparation Nathan Mahler: Acquisition of in vitro data, surgical assistant, critical review of manuscript Brad Bolon: Interpretation of ex vivo data, critical review of manuscript Andrew Niehaus: Assistance with bronchoscopy, interpretation of ex vivo data, critical review of manuscript Iyore James: Primary surgeon, acquisition of in vitro data, critical review of manuscript

Narutoshi Hibino: Primary surgeon, critical review of manuscript

Toshiharu Shinoka: Primary surgeon, critical review of manuscript

Jed Johnson: Study design, manufacture of nanofiber scaffolds, critical review of manuscript

Christopher K. Breuer: Study design and final approval of manuscript

Level of Evidence: Level II - Therapeutic Study, Prospective Cohort Study 


\begin{abstract}
:
Background: Surgical management of long segment tracheal disease is limited by a paucity of donor tissue and poor performance of synthetic materials. A potential solution is the development of a tissue-engineered tracheal graft (TETG) which promises an autologous airway conduit with growth capacity.

Methods: We created a TETG by vacuum seeding bone marrow-derived mononuclear cells (BM-MNCs) on a polymeric nanofiber scaffold. First, we evaluated the role of scaffold porosity on cell seeding efficiency in vitro. We then determined the effect of cell seeding on graft performance in vivo using an ovine model. Results: Seeding efficiency of normal porosity (NP) grafts was significantly increased when compared to high porosity (HP) grafts (NP: $360.3 \pm 69.19 \times 10^{3}$ cells $/ \mathrm{mm}^{2} ; \mathrm{HP}: 133.7 \pm 22.73 \times 10^{3}$ cells $\left./ \mathrm{mm}^{2} ; \mathrm{p}<0.004\right)$. Lambs received unseeded $(n=2)$ or seeded $(n=3)$ NP scaffolds as tracheal interposition grafts for 6 weeks. Three animals were terminated early due to respiratory complications ( $n=2$ unseeded, $n=1$ seeded). Seeded TETG explants demonstrated wound healing, epithelial migration, and delayed stenosis when compared to their unseeded counterparts.

Conclusion: Vacuum seeding BM-MNCs on nanofiber scaffolds for immediate implantation as tracheal interposition grafts is a viable approach to generate TETGs, but further preclinical research is warranted before advocating this technology for clinical application.
\end{abstract}

Keywords: Tissue engineering, trachea, electrospinning, bone marrow mononuclear cells, vacuum seeding, large animal model 


\section{Introduction ${ }^{1}$}

Current options for the management of diseases involving large segments of the upper respiratory tract such as tracheal agenesis, tracheal clefts, and tracheal stenosis are limited [1,2]. While the current standard of care involves resection of diseased airway segments with primary anastomosis, removal of more than half the tracheal length in adults or more than $1 / 3$ in children requires use of prosthetic materials. These materials lack growth potential and are fraught with complications such as stenosis, infection, rejection, and graft migration [3-5]. Donor allografts are an alternative to tracheal prostheses, but are limited by a paucity of suitable tissue, size mismatch, and the requirement for life-long immunosuppression. The application of tissue engineering technology for treatment of tracheal disease has the potential to create a fully functional tracheal conduit with growth capacity, however, mixed clinical results currently limit widespread adoption of this approach [6-9]. Early attempts with cell seeding of decellularized allografts have been recently applied with modest clinical success. Macchiarini et al. created the first tissue engineered tracheal graft (TETG) by seeding autologous mesenchymal stem cells and nasal epithelial cells onto a decellularized allograft followed by in vitro culture and implantation into the left mainstem bronchus of a 30 year old woman suffering from severe bronchomalacia [10,11]. Five-year follow-up described recurrent stenosis of the proximal anastomosis requiring multiple stents, but the remaining graft was patent and lined by a pseudostratified, ciliated columnar respiratory epithelium [12]. Similarly, Elliott et al. implanted a decelluarized tracheal allograft into a 10 year old child as an urgent compassionate-use procedure [13], bypassing in vitro culture by saturating the graft in a suspension of bone marrow mononuclear cells (BM-MNCs) at the time of surgery. Lack of epithelialization and graft laxity required stenting for two years following implantation; however, the creation of a TETG without in vitro culture was a notable accomplishment in the field.

While the progress in translation of decellularized allografts is promising, this approach is inherently limited by the availability of suitable donor tissue and the long length of time required for graft decellularization, with some cycles taking up to 20 days [9]. Active research is therefore underway to study alternative source material for TETGs, including degradable and non-degradable polymeric scaffolds, which provide a three-dimensional environment that mimics native extracellular matrix (ECM) in structure and function, allowing for cell attachment,

\footnotetext{
${ }^{1}$ Abbreviations: TETG - tissue engineered tracheal graft, BM-MNC - bone marrow mononuclear cell, NP - normal porosity, HP - high porosity, PET - polyethylene terephthalate, PU - polyurethane, SEM - scanning electron microscopy, NBF - neutral buffered formalin, H\&E - hematoxylin and eosin, ET - endotracheal, ECM extracellular matrix, PBS - phosphate buffered saline
} 
differentiation, and proliferation $[6,14]$. Recently, an electrospun polyethylene terephthalate/polyurethane (PET/PU) TETG scaffold manufactured by Nanofiber Solutions, Inc. has been seeded with autologous bone marrow-derived mononuclear cells and successfully implanted in four patients requiring tracheal replacement $[7,15]$. However, the clinical utility of this approach is limited by the requirement for cell culture in a bioreactor before implantation. Development of a seeding methodology for a TETG that bypasses in vitro culture has the potential to significantly advance this field. In the present study, we compared the efficiency of vacuum seeding BM-MNCs on this clinically utilized PET/PU TETG scaffold (defined as normal porosity, NP) to that of a high-porosity (HP) variant in order to identify a design that would provide the greatest cell density upon implantation. Next, we evaluated the effect of BM-MNC seeding on TETG performance following orthotopic implantation in a juvenile sheep model for 6 weeks.

\section{Materials and Methods}

\subsection{Animal Care / Ethics Statement}

The Institutional Animal Care and Use Committee of the Research Institute at Nationwide Children's Hospital (Columbus, $\mathrm{OH}$ ) approved and monitored all animal procedures described in the present report. All animals received humane care in compliance for the Care and Use of Laboratory Animals (2011), from the Public Health Service, National Institutes of Health (Bethesda, MD).

\subsection{Scaffold Design and Fabrication}

Tracheas from juvenile sheep $(45-60 \mathrm{~kg})$ were donated from a local abattoir (Columbus, $\mathrm{OH})$ and organ morphometry was used to inform scaffold design. Design criteria called for a D-shaped trachea a flexible dorsal ligament and C-shaped rings that mimic the native tracheal hyaline cartilage in gross morphology and mechanical stiffness. A custom mandrel was machined from stainless steel to match the dimensional measurements obtained from native juvenile sheep specimens (lumen diameter: $17 \mathrm{~mm}$ ). Polymer nanofiber precursor solutions were prepared by: 1) dissolving $8 \%$ polyethylene terephthalate (PET) in 1,1,1,3,3,3hexafluoroisopropanol (wt/wt) and heating the solution to $60^{\circ} \mathrm{C}$ followed by continuous stirring until the PET was completely dissolved, and by 2) dissolving 3\% polyurethane (PU) in 1,1,1,3,3,3-hexafluoroisopropanol $(\mathrm{wt} / \mathrm{wt}$ ) with continuous stirring at room temperature until the PU was completely dissolved. Once cooled, the solutions were combined to create a final ratio of $70 \%$ PET and 30\% PU (wt/wt). The PET/PU solution was then electrospun in a custom designed electrospinning apparatus utilizing 20 gauge blunt tip needles, a high 
voltage DC power supply set to $+14 \mathrm{kV}$, and a $15 \mathrm{~cm}$ tip-to-substrate distance. C-rings were fabricated from a medical grade polycarbonate sheet cut to the desired shape and thermo-molded around the electrospinning mandrel. The rings were then manually embedded into the graft during the process of electrospinnning and integrated within the scaffold matrix by completion of graft fabrication. All tracheal scaffolds were removed from the mandrel and placed in a vacuum overnight to ensure removal of residual solvent (typically less than 10 ppm) [16] To create the HP scaffold, salt crystals ( $0.5 \mathrm{~mm}$ particle size) were deposited onto the mandrel throughout the entire electrospinning process, followed by five $30 \mathrm{~min}$ washes in deionized water to completely dissolve the salt crystals dispersed through the construct as previously described [17]. The scaffolds were packaged in Tyvek pouches and terminally sterilized with $30 \mathrm{kGy}$ of gamma irradiation. Scaffold manufacturing methods to produce the NP scaffold replicated those used clinically and characterized by Jungebluth et al. [15]. Both NP and HP scaffold types were developed and manufactured by Nanofiber Solutions, Inc. (Columbus, OH).

\subsection{Scanning Electron Microscopy (SEM)}

Samples for SEM were cut from the tracheal scaffold and mounted on aluminum pin mounts with carbon double-sided tape. The samples were then sputter coated with a $5 \mathrm{~nm}$ thick layer of gold for observation in an FEI Nova NanoSEM 400 with an accelerating voltage of $5 \mathrm{kV}$.

\subsection{BM-MNC Isolation and Scaffold Seeding}

Bone marrow was aspirated from the iliac crests of juvenile sheep $(\mathrm{n}=6,25-45 \mathrm{~kg})$. Animals were sedated with ketamine (IV, 4 mg/kg, Patterson Veterinary), diazepam (IV, $0.5 \mathrm{mg} / \mathrm{kg}$, Patterson Veterinary), and buprenorphine (IM, $0.015 \mathrm{mg} / \mathrm{kg}$, Patterson Veterinary), anesthetized with inhaled isoflurane (Patterson Veterinary) at $0-5 \%$, vaporized with $100 \%$ oxygen at $30-60 \mathrm{~mL} / \mathrm{kg} / \mathrm{min}$ and prepared in sterile fashion. A small skin incision was made over the iliac crest, then a 15 gauge Illinois Sternal/Iliac Bone Marrow Needle (Care Express) was inserted to aspirate a maximum of $2 \mathrm{~mL} / \mathrm{kg}$ of bone marrow per animal. Syringes were heparinized with $100 \mathrm{IU} / \mathrm{mL}$ in $0.9 \%$ saline. Aspirated bone marrow was then filtered through a $100-\mu \mathrm{m}$ cell strainer (Falcon) and collected in sterile $50 \mathrm{~mL}$ conical tubes. The BM-MNC fraction was obtained from the filtered bone marrow aspirate with the Purecell Select System for Whole Blood MNC Enrichment (Pall Medical), following the manufacturer's protocol as previously described $[18,19]$. The Purecell Select System for Whole Blood MNC Enrichment (Pall Medical) has been validated by our group and is understood to yield a 
bone marrow mononuclear cell seeding suspension that is phenotypically comparable to that obtained by traditional density gradient centrifugation [18-20]. The cell suspension was subsequently vacuum seeded onto the polymeric TETG scaffolds using a previously described vacuum seeding technique (Fig 2 A) [19,21]. After seeding, TETGs were immersed in the post-seeding cell suspension $(\sim 140 \mathrm{~mL})$ and immediately delivered to the surgical suite or sampled for further analysis.

\subsection{Cell Seeding Efficiency}

Pre-seeding and post-seeding cell suspensions were collected for in-process determination of cell seeding efficiency and cell viability using a manual hemocytometer and manual Trypan blue exclusion (Life Technologies). Seeded scaffolds were sampled ( $\mathrm{n}=5$ samples per scaffold, $5 \times 5 \mathrm{~mm}$ sample) for quantitative analysis of cell attachment within the scaffold using the QuantiT $^{\mathrm{TM}}$ PicoGreen $^{\circledR}$ dsDNA assay (LifeTechnologies) following the manufacturer's protocol. One post-seeding sample from each scaffold type was fixed in neutral buffered $10 \%$ formalin (NBF) for 24 hours, routinely processed, paraffin embedded, and stained with hematoxylin and eosin (H\&E) for qualitative assessment of cell seeding efficiency.

\subsection{Surgical Implantation}

A $3.0 \mathrm{~cm}$ TETG was implanted into the mid-cervical trachea of juvenile sheep (25-45 $\mathrm{kg}, \mathrm{n}=2$ unseeded, $\mathrm{n}=3$ seeded). Under general anesthesia, a midline cervical incision was made, exposing the trachea. A $3.0 \mathrm{~cm}$ long segment of the trachea was excised. The first endotracheal (ET) tube was retracted, then a second ET tube was placed into the distal trachea to maintain ventilation. The TETG was anastomosed cranially with a 4-0 running Prolene ${ }^{\circledR}$ suture (Ethicon) and the second ET tube was removed. The first ET tube was re-advanced, and the caudal anastomosis was completed in the same fashion. Fibrin glue was applied to suture lines to ensure airtight anastomoses. Musculature, subcutis, and skin were closed in standard fashion.

\subsection{Bronchoscopy}

TETG healing was evaluated $(\mathrm{n}=1)$ at 7 and 14 days after implantation by conventional bronchoscopy. The animal was sedated with ketamine (IV, $8 \mathrm{mg} / \mathrm{kg}$, Patterson Veterinary), and diazepam (IV, $0.5 \mathrm{mg} / \mathrm{kg}$, Patterson Veterinary) and was intermittently placed on $100 \%$ oxygen $30-60 \mathrm{~mL} / \mathrm{kg} / \mathrm{min}$ by masking to maintain oxygenation. The animal was placed in sternal recumbence with neck extended and elevated by supports. A 6.6 $\mathrm{mm}$ diameter x $1.0 \mathrm{~m}$ long flexible direct view endoscope (VFS-2B, VetVu) attached to a halogen light source (VetVu 1185U) was advanced through the oropharynx and guided through the cervical trachea and TETG. The 
luminal tissue of the TETG was sampled (mid-graft) with an Alligator jaw cup (VetVu). Cytologic samples were placed on polarized glass slides (Tissue Path, Fisherbrand), dried, and stained with Wright-Giemsa (Astral Diagnostics) following the manufacturer's protocol.

2.8 Tissue Harvest, Processing, Histology, and Immunostaining

At the study endpoint, sheep were deeply sedated with telazol (IV, $7.5 \mathrm{mg} / \mathrm{kg}$, Patterson Veterinary) and ketamine (IV, $20 \mathrm{mg} / \mathrm{kg}$, Patterson Veterinary) and euthanized by induction of pneumothorax with bilateral thoracic incisions. Animals were autopsied and the TETGs explanted. Tissue and graft samples were placed in 10\% NBF (Fisher) for 48-72 hours, trimmed, and submitted for routine histological processing and paraffin embedding with hard wax (Richard Allan Paraffin Type 9, ThermoScientific). $4 \mathrm{~m}$ thick sections were stained with H\&E. Immunostaining was performed to identify neovascularization of the TETG at 6 weeks. Briefly, sections were deparaffinized, rehydrated, and antigens were retrieved with citrate buffer (Target Retrieval Solution, Dako, Carpinteria, CA). Samples were blocked for endogenous peroxidase activity (IHC only) and nonspecific background staining before incubation with the following primary antibodies: mouse anti-human smooth muscle actin (Dako, 1:100), rabbit anti-human Factor VIII (Dako, 1:200), and mouse anti-human vWF (Dako, 1:1000). Chromogenic detection was performed by incubation with species appropriate biotinylated secondary antibodies followed by the Vector RTU Elite Complex and development with 3, 3'-

Diaminobenzidine (DAB, Vector). Fluorescent detection was performed by incubation with anti-mouse Alexafluor 488 (1:300, Life Technologies, Grand Island, NY). Cell nuclei were identified by counterstaining with either hematoxylin (Vector) or 4',6-diamidino-2-phenylindole (DAPI, Slowfade Gold, Life Technologies) and cover slipped. Photomicrographs were obtained with a Zeiss Axio Observer Z.1.

\subsection{Statistical Analysis}

Data were collected prospectively and statistically analyzed with Graphpad Prism software using unpaired, twotailed student's t-tests. Data are reported as mean and standard error of the mean and were considered significant if $\mathrm{p}<0.05$.

\section{Results}

3.1 Electrospun TETG scaffolds mimic native tracheal anatomy TETG scaffolds closely mimicked native juvenile sheep tracheal anatomy and featured cartilage-like C-rings embedded within the polymer construct and a flexible dorsal ligament (Figure 1A, D). Qualitative comparison 
of NP (Fig. 1 B-C) and HP (Fig. 1 E-F) scaffolds with SEM revealed that salt elution was a successful technique to increase void space between electrospun PET/PU nanofibers (i.e. porosity).

3.2 Vacuum seeding of BM-MNCs creates TETG without in vitro culture

BM-MNCs were successfully isolated from the iliac crests of juvenile sheep and yielded $5.9 \times 10^{10} \pm 4.2 \times 10^{10}$ total cells from $0.5-2.0 \mathrm{~mL} / \mathrm{kg}$ of body weight. Seeding efficiency ranged between $37-75 \%(\mathrm{n}=6,61 \pm 5.4 \%)$ for NP grafts and $20-29 \%(n=3,23 \pm 3.0 \%)$ for HP grafts $(* * p<0.002$; Fig. 2D). Histology of formalin-fixed paraffin embedded samples from seeded scaffolds demonstrated mixed cellular infiltrates (myeloid and erythroid lineages by histomorphology) embedded and interspersed throughout the polymeric fibers of both NP and HP scaffolds (Fig. 2B, C). DNA quantification was performed with graft samples frozen immediately postseeding to ensure no cell proliferation or disassociation from the scaffold. NP grafts demonstrated significantly more cells $/ \mathrm{mm}^{2}$ than HP grafts ( $\mathrm{n}=3 /$ group; $360.3 \pm 69.19 \times 10^{3}$ vs. $133.7 \pm 22.73 \times 10^{3}$ cells $/ \mathrm{mm}^{2} ; * * \mathrm{p}<0.004$ Fig. 2E).

3.3 BM-MNC Seeded TETGs function as a respiratory conduit for 6 weeks

Due to the superior performance of the normal porosity graft in comparison to the high porosity graft in terms of cell seeding efficiency and total seeded cell number, we selected the normal porosity scaffold for implantation into a juvenile sheep model to elucidate the effect of cell seeding on TETG performance in vivo. Seeded and unseeded NP TETGs were implanted into juvenile sheep (seeded: $n=3$; unseeded: $n=2$ ). Mean operative time was two hours and operative mortality was $0 \%$ (0/5 animals). At days 7 and 14 after implantation, one animal underwent bronchoscopy to monitor TETG function. Wright-Giemsa stained cytologic samples of biopsy tissue acquired from the lining of the TETG lumen at day 14 demonstrated epithelial cells, macrophages, and neutrophils consistent with neoepithelialization and an ongoing sub-acute inflammatory response (Fig. 3A). Three animals required early euthanasia due to respiratory failure (increased respiratory effort, tachypnea, stridor, and hypoxia) due to graft stenosis at day 34 (seeded: $\mathrm{n}=1$ ), day 36 , and day 40 (unseeded: $\mathrm{n}=2$ ). In correlating cell-seeding data from implanted scaffold samples to implant performance, the scaffold of the animal from the seeded group requiring early euthanasia (day 34) was that with the lowest cell seeding density of all seeded scaffolds $\left(2.0 \times 10^{4}\right.$ cells $\left./ \mathrm{mm}^{2}\right)$. The only animals to survive until the 6 -week study endpoint were those receiving BM-MNC seeded TETGs ( $\mathrm{n}=2$ ). Upon TETG explant, all animals demonstrated mild to moderate stenosis at the level of the proximal and distal anastomoses (Fig. 3B). This 
stenosis manifested as respiratory distress at the 6-week time point in the seeded grafts. No other gross organ pathology was identified at autopsy that was attributed to TETG implantation.

Histologic analysis of TETG explants from both the seeded and unseeded groups demonstrated granulation tissue comprised of cellular fibrous tissue containing extensive extracellular matrix deposition, many new blood vessels surrounding and overlying the graft, and a hyperplastic native mucosal epithelium containing a mixed inflammatory cell infiltrate. TETG lumens were occasionally noted to contain serocellular debris and mixed bacteria. In the seeded group, an epithelial migration front was identified transitioning from the native mucosal surface at the margins of the anastomoses over the granulation tissue above and below the graft (Fig. 3C). Immunohistochemical and immunofluorescent staining for markers of endothelial (Factor VIII and vWF) and vascular smooth muscle cells ( $\alpha$-smooth muscle actin) were performed and demonstrated abundant vascular profiles underlying the neo-epithelium positive for both endothelial cells and vascular smooth muscle cells (Fig. 4).

\section{Discussion}

Advances in tissue engineering technology offer potential solutions to these challenges with the goal of developing a readily available autologous tracheal conduit with ability to grow, support the respiratory demands of the host, and self-repair over the course of a patient's life. To successfully translate a tissue engineered trachea from the bench to the bedside, appropriate preclinical investigation is vital to ensure both safety and efficacy.

In this work, we aimed to support and advance the preclinical validation of the PET/PU electrospun scaffold by confirming the efficacy of a vacuum seeding technique and developing a juvenile sheep model to evaluate the effect of BM-MNC cell-seeding on TETG performance. Vacuum seeding of these scaffolds with an autologous BM-MNC fraction isolated via a closed, disposable system demonstrated that the NP PET/PU scaffold best supported cell engraftment and resulted in the greatest efficiency of cell seeding in comparison to its highporosity counterpart. Since the HP scaffold yielded a significantly lower seeding efficiency, and the objective of our study was to investigate the effect of cell seeding on neotissue development in a TETG, we selected the NP scaffold for in vivo application. However, it may be that the HP scaffold, while not able to retain seeded cells with the same efficiency as the NP scaffold, may better allow for autologous cell infiltration and tissue engraftment. We suggest that the NP and HP scaffold lie on two ends of a spectrum within which is found the ideal TETG scaffold design, 
allowing for the synergistic contributions of cell seeding and autologous cell infiltration all while maintaining a viable airway for the host over the course of neotissue formation.

Implantation of seeded and unseeded NP scaffolds in a large animal model was successful and no acute TETG failure was observed, but respiratory failure due to graft stenosis resulted in euthanasia before the study endpoint in 3/5 animals: $2 / 2$ unseeded scaffolds and 1/3 seeded scaffolds. It is plausible that the observed seeded TETG failure is due to a relatively low cell seeding density in this animal, suggesting that BM-MNC seeding may improve TETG function in a dose dependent manner and points to the importance of developing proper in-process release criteria to meet an acceptable minimum cell seeding threshold that will support TETG function and neotrachea development.

\section{Conclusion}

The results of this pilot study suggest that the use of a culture-free, vacuum seeding protocol after BMMNC isolation with a closed, disposable system is appropriate for electrospun polymeric tracheal grafts in a large animal model. The elimination of in vitro culture is a significant advancement for translation of the TETG to the clinic, and this work clearly demonstrates the clinical potential of using a vacuum seeding system immediately before implantation. These TETGs can successfully function as airway conduits for up to 6 weeks post-operatively, but are characterized by the development of stenosis at the sites of anastomosis. Further preclinical research to optimize scaffold design and cell seeding dose is warranted before pursuing clinical application.

\section{Acknowledgements}

The work presented was supported by the National Science Foundation Award \# IIP-1315524 (Nanofiber Solutions, Inc.) and T32 OD010429-13 (ESC). The Comparative Pathology and Mouse Phenotyping Shared Resource, Department of Veterinary Biosciences and the Comprehensive Cancer Center is supported in part by grant P30 CA016058. The Morphology Core at Nationwide Children's Hospital Tissue performed tissue processing and $\mathrm{H} \&$ E staining. Specifically, we would like to thank Dr. Nina Zitzer, the NCH Animal Care staff, Yongjie Miao (Biostatistician for the NCH Cardiovascular and Pulmonary Research Institute), Matt Wiet, Ben Brownback and Josh Meeter (B2 Group) for their assistance. 


\section{References}

[1] Austin J, Ali T. Tracheomalacia and bronchomalacia in children: pathophysiology, assessment, treatment and anaesthesia management. Paediatr Anaesth 2003;13:3-11. doi:10.1046/j.1460-9592.2003.00802.x.

[2] Antón-Pacheco JL, Cano I, García A, Martínez A, Cuadros J, Berchi FJ. Patterns of management of congenital tracheal stenosis. J Pediatr Surg 2003;38:1452-8. doi:10.1016/S0022-3468(03)00495-0.

[3] Kojima K, Vacanti CA. Tissue engineering in the trachea. Anat Rec (Hoboken) 2014;297:44-50. doi:10.1002/ar.22799.

[4] Kucera KA, Doss AE, Dunn SS, Clemson LA, Zwischenberger JB. Tracheal replacements: part 1. ASAIO J 2007;53:497-505. doi:10.1097/MAT.0b013e3180616b5d.

[5] Doss AE, Dunn SS, Kucera KA, Clemson LA, Zwischenberger JB. Tracheal replacements: Part 2. ASAIO J 2007;53:631-9. doi:10.1097/MAT.0b013e318145ba13.

[6] Fishman JM, Wiles K, Lowdell MW, De Coppi P, Elliott MJ, Atala A, et al. Airway tissue engineering: an update. Expert Opin Biol Ther 2014;14:1477-91. doi:10.1517/14712598.2014.938631.

[7] Jungebluth P, Macchiarini P. Airway transplantation. Thorac Surg Clin 2014;24:97-106. doi:10.1016/j.thorsurg.2013.09.005.

[8] Crowley C, Birchall M, Seifalian AM. Trachea transplantation: from laboratory to patient. J Tissue Eng Regen Med 2014. doi:10.1002/term.1847.

[9] Jungebluth P, Alici E, Baiguera S, Le Blanc K, Blomberg P, Bozóky B, et al. Tracheobronchial transplantation with a stem-cell-seeded bioartificial nanocomposite: a proof-of-concept study. Lancet 2011;378:1997-2004. doi:10.1016/S0140-6736(11)61715-7.

[10] Macchiarini P, Jungebluth P, Go T, Asnaghi MA, Rees LE, Cogan TA, et al. Clinical transplantation of a tissue-engineered airway. Lancet 2008;372:2023-30. doi:10.1016/S0140-6736(08)61598-6.

[11] Bader A, Macchiarini P. Moving towards in situ tracheal regeneration: the bionic tissue engineered transplantation approach. J Cell Mol Med 2010;14:1877-89. doi:10.1111/j.1582-4934.2010.01073.x.

[12] Gonfiotti A, Jaus MO, Barale D, Baiguera S, Comin C, Lavorini F, et al. The first tissue-engineered airway transplantation: 5-year follow-up results. Lancet 2014;383:238-44. doi:10.1016/S0140-6736(13)62033-4.

[13] Elliott MJ, De Coppi P, Speggiorin S, Roebuck D, Butler CR, Samuel E, et al. Stem-cell-based, tissue engineered tracheal replacement in a child: a 2-year follow-up study. Lancet 2012;380:994-1000. doi:10.1016/S0140-6736(12)60737-5.

[14] Teoh GZ, Crowley C, Birchall MA, Seifalian AM. Development of resorbable nanocomposite tracheal and bronchial scaffolds for paediatric applications. Br J Surg 2015;102:e140-50. doi:10.1002/bjs.9700.

[15] Jungebluth P, Haag JC, Lim ML, Lemon G, Sjöqvist S, Gustafsson Y, et al. Verification of cell viability in bioengineered tissues and organs before clinical transplantation. Biomaterials 2013;34:4057-67. doi:10.1016/j.biomaterials.2013.02.057. 
[16] Nam J, Huang Y, Agarwal S, Lannutti J. Materials selection and residual solvent retention in biodegradable electrospun fibers. J Appl Polym Sci 2008;107:1547-54. doi:10.1002/app.27063.

[17] Nam J, Huang Y, Agarwal S, Lannutti J. Improved Cellular Infiltration in Electrospun Fiber via Engineered Porosity. Tissue Eng 2007;13:2249-57. doi:10.1089/ten.2006.0306.

[18] Kurobe H, Tara S, Maxfield MW, Rocco KA, Bagi PS, Yi T, et al. Comparison of the Biological Equivalence of Two Methods for Isolating Bone Marrow Mononuclear Cells for Fabricating TissueEngineered Vascular Grafts. Tissue Eng Part C Methods 2014. doi:10.1089/ten.TEC.2014.0442.

[19] Kurobe H, Maxfield MW, Naito Y, Cleary M, Stacy MR, Solomon D, et al. Comparison of a closed system to a standard open technique for preparing tissue-engineered vascular grafts. Tissue Eng Part C Methods 2015;21:88-93. doi:10.1089/ten.TEC.2014.0160.

[20] Hibino N, Nalbandian A, Devine L, Martinez RS, McGillicuddy E, Yi T, et al. Comparison of human bone marrow mononuclear cell isolation methods for creating tissue-engineered vascular grafts: novel filter system versus traditional density centrifugation method. Tissue Eng Part C Methods 2011;17:993-8. doi:10.1089/ten.TEC.2011.0110.

[21] Udelsman B, Hibino N, Villalona G a, McGillicuddy E, Nieponice A, Sakamoto Y, et al. Development of an operator-independent method for seeding tissue-engineered vascular grafts. Tissue Eng Part C Methods 2011;17:731-6. doi:10.1089/ten.tec.2010.0581. 


\section{Figure Legends}

1. Scaffold Characterization: Normal porosity (NP) tracheal scaffolds were electrospun from PET/PU and embedded with 3 cartilaginous " $C$ " rings (A). SEM of the cross section (B) and surface (C) of the NP grafts. High porosity (HP) scaffolds were made in the same manner with salt crystals embedded within stratum of the graft and eluted after spinning (D). SEM of the cross section (E) and surface (F) of the HP grafts demonstrate increased void space between nanofibers as a result of salt-elution; fiber diameter in both grafts is comparable.

2. TETG Seeding: (A) Schematic of closed-disposable vacuum seeding apparatus. Filtered bone marrow aspirate (a) is passed through the "Leukoharvest" filter (b), erythrocytes, serum, and plasma are collected in (c), elution solution (d) flushes filtered BM-MNC into the seeding chamber (e) containing PBS, a fenestrated mandrel, and the TETG scaffold affixed with Tegaderm ${ }^{\circledR}$. The vacuum regulator (f) induces negative pressure to draw the cell solution through the graft and mandrel for collection into specimen traps (g). Photomicrographs of H\&E stained NP (B) and HP (C) grafts after vacuum seeding. Seeding efficiency was assessed both in-process and post-process by manual hemocytometer counts (D) and DNA assay (E) respectively.

3. Post-implantation evaluation: (A) Photomicrograph of Wright-Giemsa stained cytologic smear from tissue lining the TETG lumen demonstrating epithelial cells (black arrows) and macrophages (open arrows) at 14 days post-implantation; Bar $=20 \mu \mathrm{m}$. (B) Gross photograph of the proximal anastomosis of a 6-week NP TETG explant demonstrating neotissue formation and stenosis. (C) Photomicrograph of H\&E stained neotissue at the level of the proximal anastomosis demonstrating mature epithelium (E) and granulation tissue overlying the TETG scaffold (G); (S) denotes suture; Bar $=500 \mu \mathrm{m}$.

4. Neovascularization: IHC for Factor VIII (A) and $\alpha$-smooth muscle actin (B) demonstrating the intimal endothelial cell and medial smooth muscle cell layers of neovasculature in the TETG. Immunofluorescent staining for vWF (green) and nuclei (DAPI) similarly demonstrate the endothelial cell layer of these neovascular profiles $(\mathrm{C})$. Scale bars $=50 \mu \mathrm{m}$, inset scale bar $=10 \mu \mathrm{m}$. 
1
2
3
4
5
6

Fig. 1

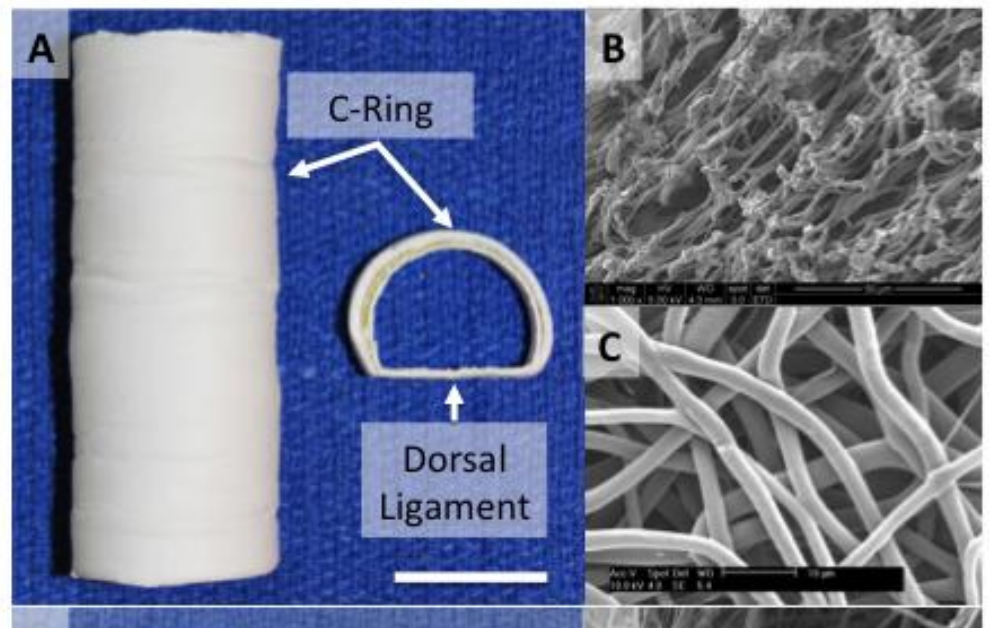

7
8
9 
Figure 2

1
2
3
4
5
6
7
8

Fig. 2

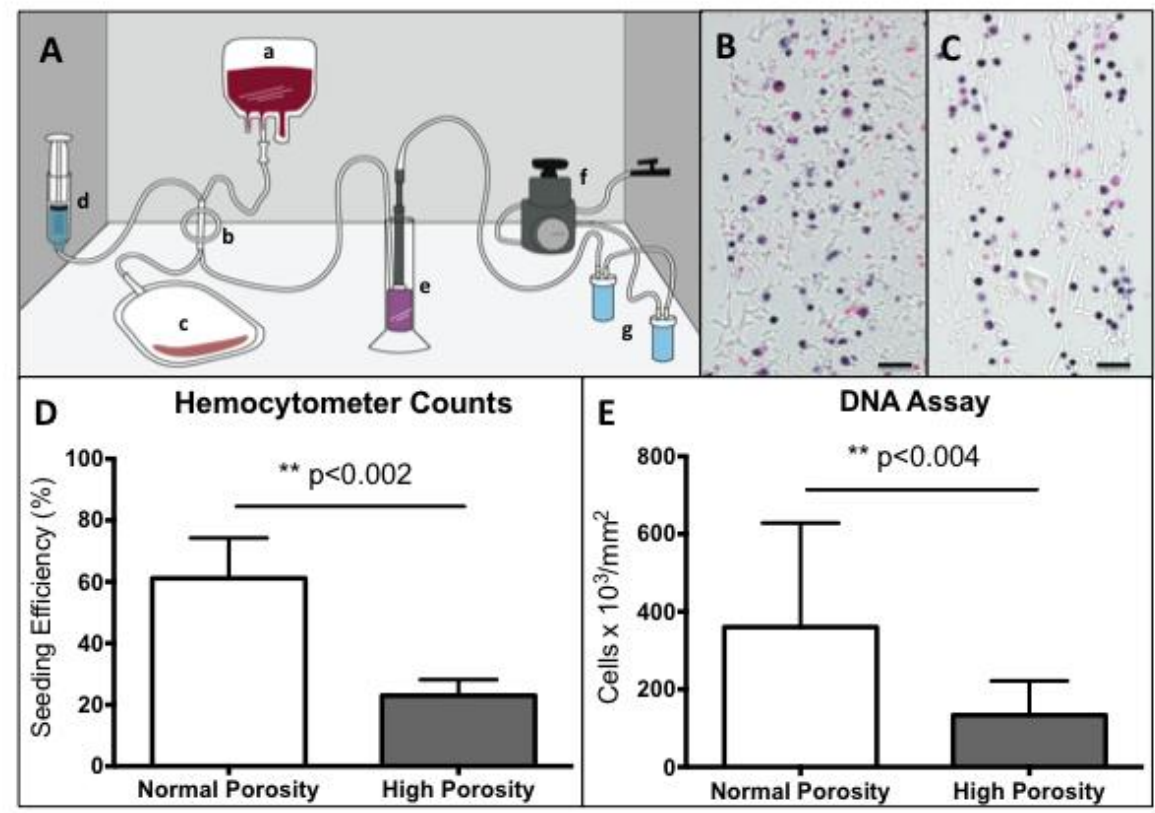

10 


1
2
3
4
5
6
7
8
9
10
11
12

Fig. 3

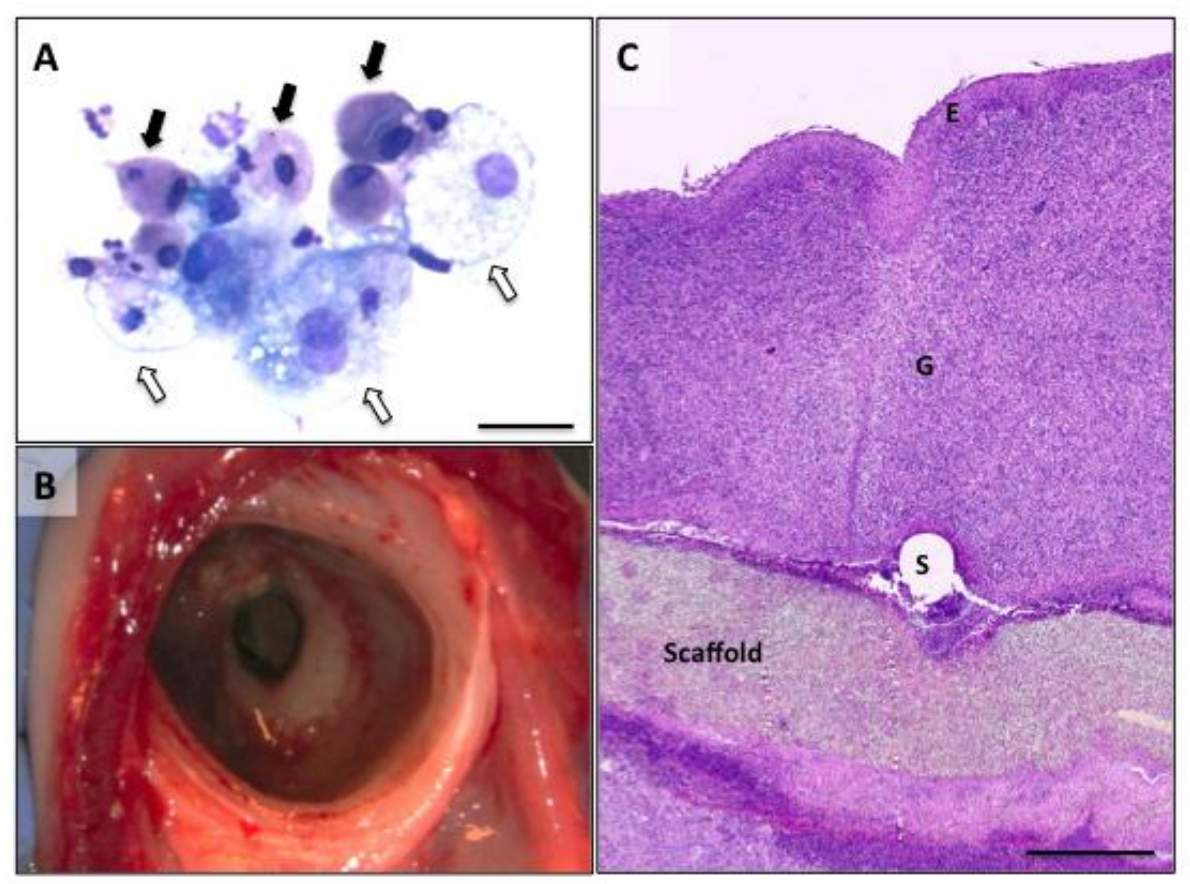

13 
Fig. 4

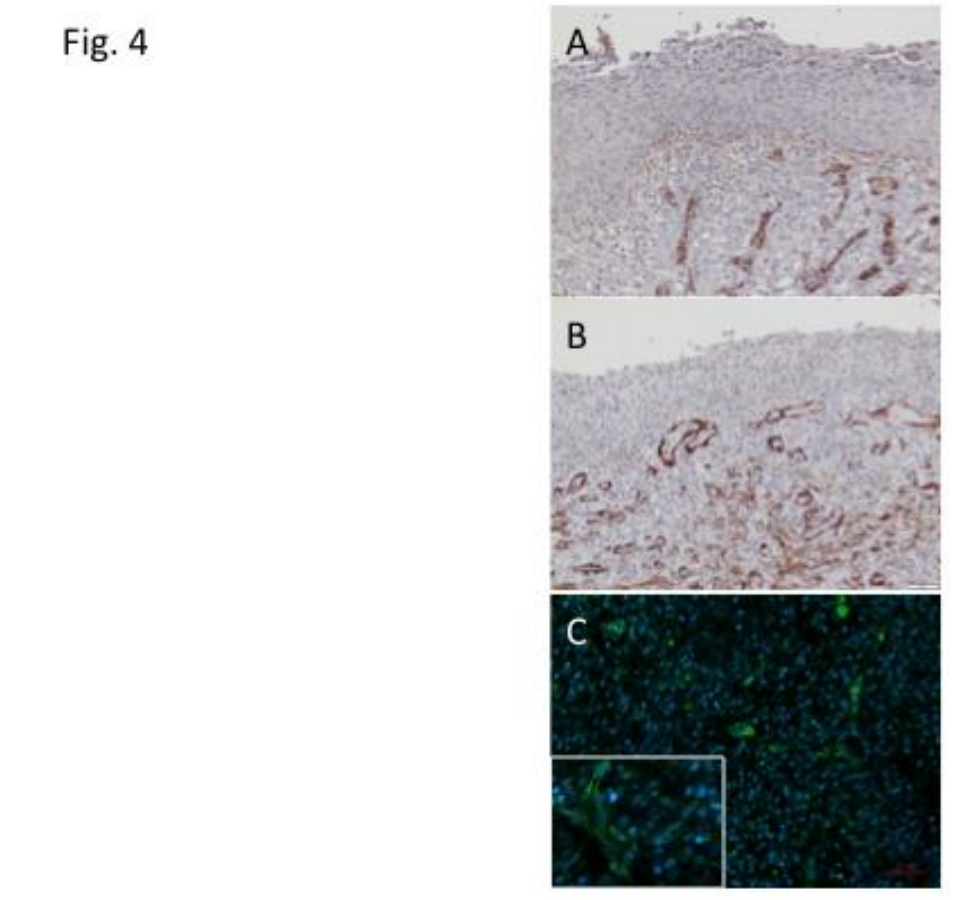

Figure 4

1

\section{.}

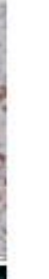

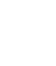

Figure 4

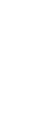

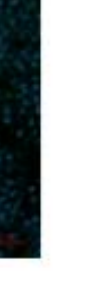

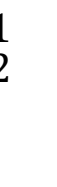

\section{列}

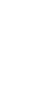

